\title{
Lamellar hole-associated epiretinal membrane is a common feature of macular holes in retinitis pigmentosa
}

\author{
Jingjing Liu ${ }^{1} \cdot$ Jiao Lyu ${ }^{1} \cdot$ Xiang Zhang ${ }^{1} \cdot$ Peiquan Zhao ${ }^{1}$
}

Received: 11 February 2019 / Revised: 11 May 2019 / Accepted: 16 July 2019 / Published online: 27 August 2019

(c) The Author(s), under exclusive licence to The Royal College of Ophthalmologists 2019

\begin{abstract}
Objective To describe the features and surgical outcomes of macular holes (MHs) in patients with retinitis pigmentosa (RP). Methods A review of consecutive series of 110 patients (206 eyes) with RP who underwent comprehensive ophthalmic examinations was conducted. Eleven eyes of ten RP patients were identified with MHs (full thickness or lamellar). Atypical epiretinal membrane, which appeared on spectral-domain optical coherence tomography (SD-OCT) images as a thick homogenous layer of moderately reflective material that was present on the inner retinal layer, was considered to be lamellar hole-associated epiretinal proliferation (LHEP). Five eyes underwent modified vitreoretinal surgery, where hole margin LHEP tissue was retained.

Results Nine eyes exhibited a lamellar macular hole (LMH), one exhibited a full-thickness macular hole (FTMH), and one exhibited both FTMH and LMH. LHEP was found in all eyes, identified intraoperatively as yellowish, sticky epiretinal membrane with internal limiting membrane beneath it. Two eyes experienced spontaneous closure of MHs without visual acuity (VA) improvement. Five eyes that underwent surgery achieved sealed MHs post-operatively and demonstrated improved, but limited, vision at their latest follow-up.

Conclusions LHEP is common in MHs associated with RP. While some eyes could achieve spontaneous closure without any VA changes, a conservative vitreoretinal surgery approach, in which the hole margin LHEP tissue is spared, can effectively repair these MHs with limited VA improvement.
\end{abstract}

\section{Introduction}

Retinitis pigmentosa (RP) includes a wide range of hereditary retinal degenerative diseases that affect the outer retina. The prevalence of RP was estimated to be 1/4000 in a world-wide population [1] and it is classically characterized by the presence of several phenomena: pale optic disc; attenuation of the retinal vessels; a typical retinal bone spicule pigmentary change involving the equatorial retina;

Prior presentation: None.

Supplementary information The online version of this article (https:// doi.org/10.1038/s41433-019-0563-3) contains supplementary material, which is available to authorized users.

Peiquan Zhao

zhaopeiquan@xinhuamed.com.cn

1 Department of Ophthalmology, Xin Hua Hospital, Affiliated to Shanghai Jiao Tong University School of Medicine,

Shanghai 200092, China nyctalopia; and progressive contraction of the visual field. Central vision is usually spared until comparatively late in the disease, or when macular complications occur, such as cystoid macular oedema (CMO), epiretinal membrane (ERM), or formation of a macular hole (MH) [2].

High-resolution spectral-domain optical coherence tomography (SD-OCT) provides detailed information about structural changes in the macular area, which facilitates better understanding of the pathogenesis of various macular lesions. Lamellar hole-associated epiretinal proliferation (LHEP) is a newly identified OCT phenomenon that consists of a thick layer of moderately reflective material that fills the space between the inner border of the ERM and the retinal nerve fibre layer. This layer is non-contractile and found predominantly in cases of lamellar MH (LMH) [3].

The formation of MHs is infrequent in RP patients; thus, clinical features of these eyes are not well known. However, MHs may represent more severe cases of macular lesion formation and are suspected to occur secondary to deroofing of coalescent macular cysts and contraction of ERM $[4,5]$. Previous studies have shown that the presence of LHEP is 
associated with more severe macular lesions and with outer retinal disruption [6-8], which are similar to conditions found in RP patients. However, the occurrence of this unique epiretinal proliferation has not yet been studied in RP who exhibit MHs. In addition, surgical interventions for the condition remain controversial. Whereas some reports have described functional and morphological improvements after concurrent vitrectomy and internal limiting membrane (ILM) peeling [2, 9, 10], other studies have concluded that surgical intervention is not recommended because spontaneous closure has been observed in some patients [11]. Therefore, the purpose of this study was to explore LHEP and other anatomical features (using SD-OCT) and report on surgical findings of MHs in RP patients.

\section{Methods}

We retrospectively reviewed a consecutive series of 110 patients (206 eyes) with RP. All patients presented to the eye clinic at Shanghai Jiao Tong University School of Medicine Xin Hua Hospital between January 2010 and October 2018 and were treated and followed by a single ophthalmologist (PZ). Diagnosis of RP was based on the presence of the following clinical signs in a complete ophthalmological examination [including measurement of best-corrected visual acuity and intraocular pressure, slit-lamp biomicroscopy, dilated fundus examination, full-field electroretinograms, and SD-OCT]: hallmark symptoms, such as night blindness and increasing visual field constriction; attenuated retinal vessels; retinal pigment epithelium granularity and mottling; bone spicule intraretinal pigmentation; optic nerve head pallor; and photoreceptor dysfunction. From among the RP patients in this study, we identified a subgroup of 11 eyes of ten patients that exhibited formation of MHs upon evaluation by SD-OCT (RTVue-100, Optovue Inc, Fremont, CA). LMH was diagnosed based on the following OCT findings: presence of irregular foveal contour, presence of a break in the inner fovea, separation of layers in the neurosensory retina, and an absence of full-thickness foveal defects. A distinctive OCT finding, a thick layer of moderately reflective material without any contractive properties on the epiretinal surface, was considered to be the lamellar hole-associated ERM (LHEP).

Demographic and clinical features, as well as OCT findings, were documented for these patients. Five eyes of five patients underwent surgical repair of MHs after a comprehensive risk/benefit evaluation and subsequent discussion with each patient. The common surgical procedure included three-port pars plana vitrectomy, ILM peeling (ILMP), and perfluoropropane gas tamponade. If LHEP tissue (sticky yellowish epiretinal proliferation) was observed during operation, it was peeled with end-gripping forceps. However, it was not forcefully removed from the hole margin; a vitreous cutter was used to trim it to the appropriate size, then it was introduced to the MH (Supplemental video). Concomitant cataract operation was performed when a combined procedure was planned. Preoperative and postoperative clinical findings were recorded and analysed.

The study was conducted in compliance with the Declaration of Helsinki and was approved by the Institutional Review Board of Xinhua Hospital Affiliated to Shanghai Jiao Tong University School of Medicine. Informed consent was obtained from all patients.

\section{Results}

Using OCT imaging, MHs were detected in 11 eyes (5.3\%) of ten patients (seven men and three women): one exhibited full-thickness MH (FTMH), nine exhibited LMH, and one exhibited both FTMH and LMH. The mean age of the patients was $50.1 \pm 11.9$ years (mean \pm standard deviation; range 34-67). The clinical characteristics of the patients are shown in Table 1.

\section{SD-OCT findings}

LHEP was detected in all 11 eyes $(100 \%)$ that exhibited MHs (Fig. 1). Two eyes with FTMH (100\%) exhibited complications of macular retinal detachment. In two eyes with LMH (20\%), associated CMO was found on OCT. The presence of a foveal bump was noted in six LMH eyes (60\%). Further, five eyes $(83.3 \%$ ) with a foveal bump demonstrated a combination of wide intraretinal defects. One eye with LMH (10\%) was complicated with extensive and highly elevated macular retinoschisis. Simultaneous condensed posterior hyaloid and vitreoretinal adhesion were detected in one eye with LMH (10\%). ERM was also noted in one $\mathrm{LMH}$ eye $(10 \%)$.

\section{Surgical outcomes}

Five eyes demonstrated severely vision disturbing conditions such as retinal detachment, highly elevated retinoschisis, ERM, and CMO were offered surgery. All of them achieved closure of the MHs (100\%) (Fig. 2) and demonstrated limited postoperative improvements in visual acuity (VA). No patients experienced reopening of the MHs during follow up. During operative procedures, the presence of LHEP was confirmed: a yellow-coloured sticky tissue that could not easily be removed from the retinal surface. Notably, LHEP is quite different from traditional ERM, which is semi-transparent, rigid, and easy to grasp. The LHEP was trimmed and introduced into the MH (Supplementary video). 


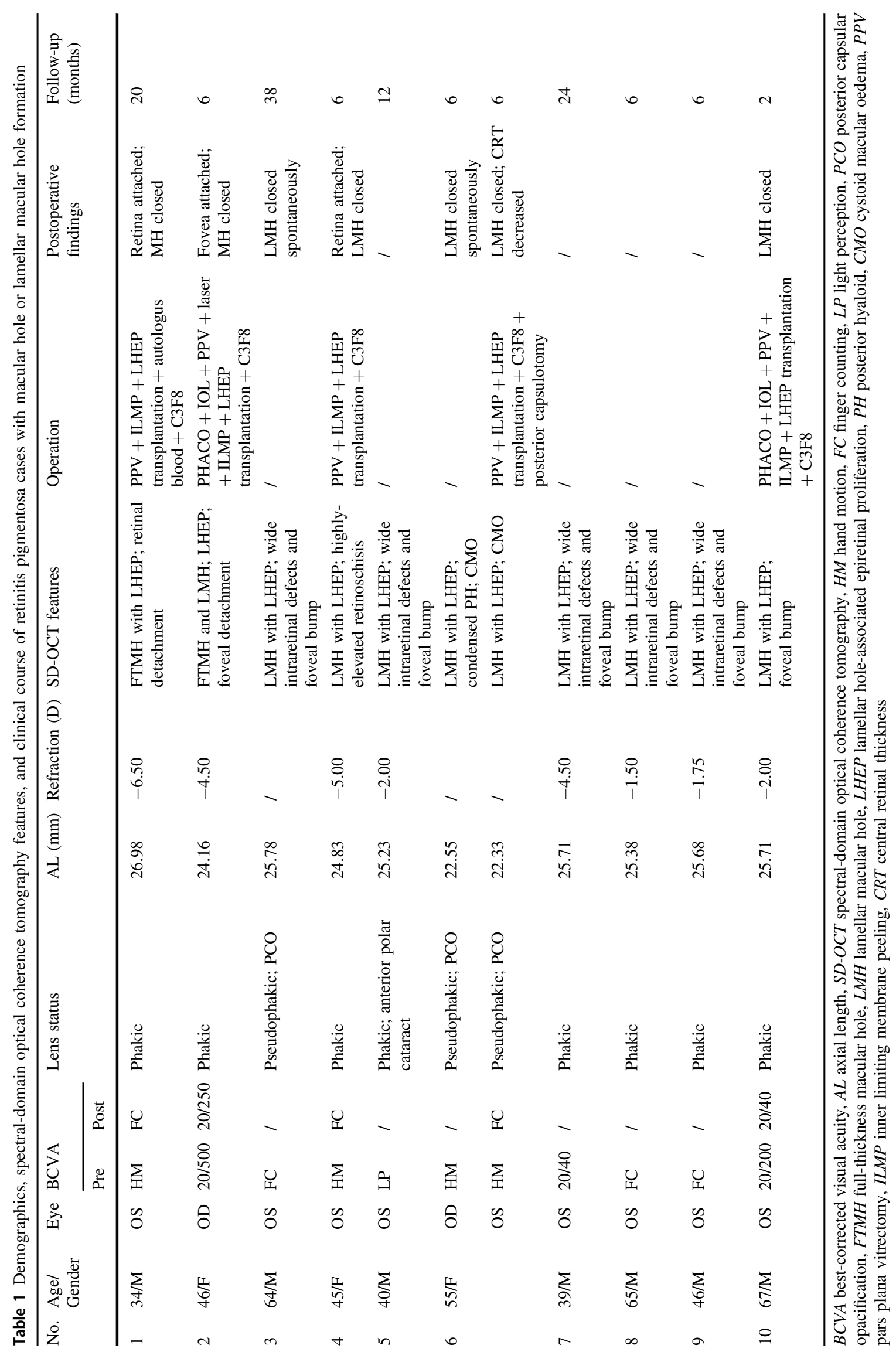



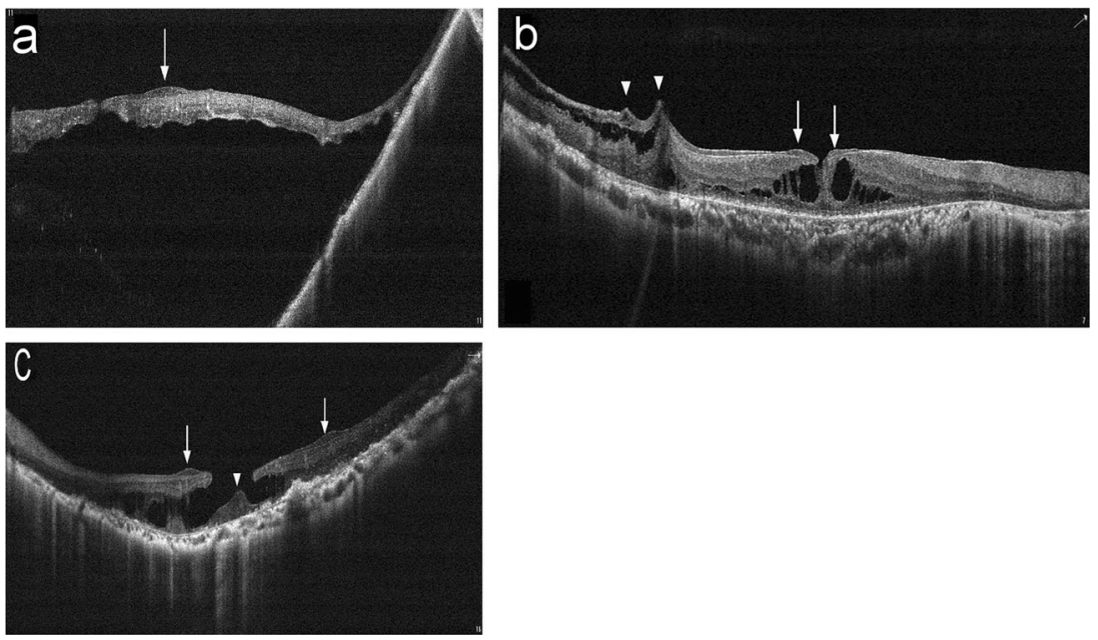

Fig. 1 Representative optical coherence tomography (OCT) images of lamellar hole-associated epiretinal membrane (LHEP) in macular holes in retinitis pigmentosa patients. a OCT scan shows a macular hole accompanied by macular detachment in the left eye of patient 1 . LHEP appeared on OCT images as a thick, homogenous layer of moderately reflective material, present on the inner retinal layer (arrow). b OCT

\section{Spontaneous closure}

Two eyes experienced spontaneous closure during follow up (Fig. 3). However, no improvements in VA were documented.

\section{Discussion}

The formation of MHs in RP patients has not frequently been reported and most were case reports [2, 5, 9, 12,13]. Therefore, clinical features of these eyes are not fully described. In our cohort, 5.3\% of RP patients had developed MHs. We used high-resolution SD-OCT to study these eyes. Notably, we found that all study eyes demonstrated LHEP on OCT imaging. LHEP is an atypical epiretinal proliferation without tractional properties. It was first reported in 2006 by Witkin et al. [14]. and later described as LHEP by Pang et al. [15]. Upon immunohistochemical analysis, LHEP tissue was reported to consist mainly of hyalocytes and fibroblasts [16]. In 2002, Amemiya et al. [17]. also found a thick yellow ERM around the MH in one eye from a patient with RP; this membrane was removed during surgery. Upon histology and cytology analysis, the removed tissue was found to contain macrophages, Müller cells, glial cells, and fibroblasts, along with many granules that appeared to be xanthophyll pigments located outside cell bodies. According to this description, the thick ERM in that report was LHEP. The high prevalence of LHEP observed here in MHs from RP patients may be associated with image of patient 6 shows a lamellar macular hole (LMH) with cystoid macular oedema (CMO) in the right eye; LHEP (arrows) was fused with condensed posterior cortical vitreous (arrowheads). Vitreoretinal traction is apparent. c OCT image of patient 5 demonstrates the LMH with LHEP (arrows); presence of a foveal bump (arrowhead) and wide intraretinal defects were noted

progressive retinal tissue loss, which has been described as an LHEP risk factor [6]. LHEP has been proposed as a Müller cell-driven healing process that originates from the inner retinal layers of the macular defect [15]. Therefore, severe disruption of retinal tissue in MH eyes with RP may stimulate Müller cell proliferation and LHEP formation, which contributes to the high prevalence of LHEP in these eyes.

Operation was performed in five eyes. Among these eyes, highly elevated macular retinoschisis was found in one eye with myopia on OCT (patient no. 4). Though the axial length was not excessive, ultrasonography showed posterior staphyloma in this eye. The formation of retinoschisis in this eye might have been caused by globe stretching in the staphyloma area [18]. Previous study also found that macular retinoschisis could not only occur in eyes that were highly myopic or with an excessive axial length but also in those with only a posterior staphyloma [19]. Resolved retinoschisis and sealed MH were found on postoperative OCT. Retinal detachment in the macular region was found in two eyes of two patients (patient no. 1 and 2) on OCT analysis. We speculate that the macular detachment in patient no. 1 developed secondary to macular retinoschisis, as OCT scans showed a detached neuroretinal layer with a ragged outer nuclear layer and a retinal split near the demarcation line. The formation of macular retinoschisis in this eye might have been caused by globe elongation in a highly myopic eye.

A previous study showed that high myopia is more prevalent in eyes that exhibit FTMH and LHEP, compared with eyes that exhibit FTMH and no LHEP [20]. Highly myopic 
Fig. 2 a Ultra-wide scanning laser ophthalmoscopy (UWSLO) demonstrates peripheral salt-pepper like pigmentation in the left eye of patient 2. b, c Spectral-domain optical coherence tomography (SD-OCT) images show both full-thickness macular hole (MH) and lamellar $\mathrm{MH}$ in the left eye. Lamellar holeassociated epiretinal membrane (LHEP) (arrow) and foveal detachment were also detected. d Postoperative OCT image shows the MHs were closed and the fovea was attached in the left eye
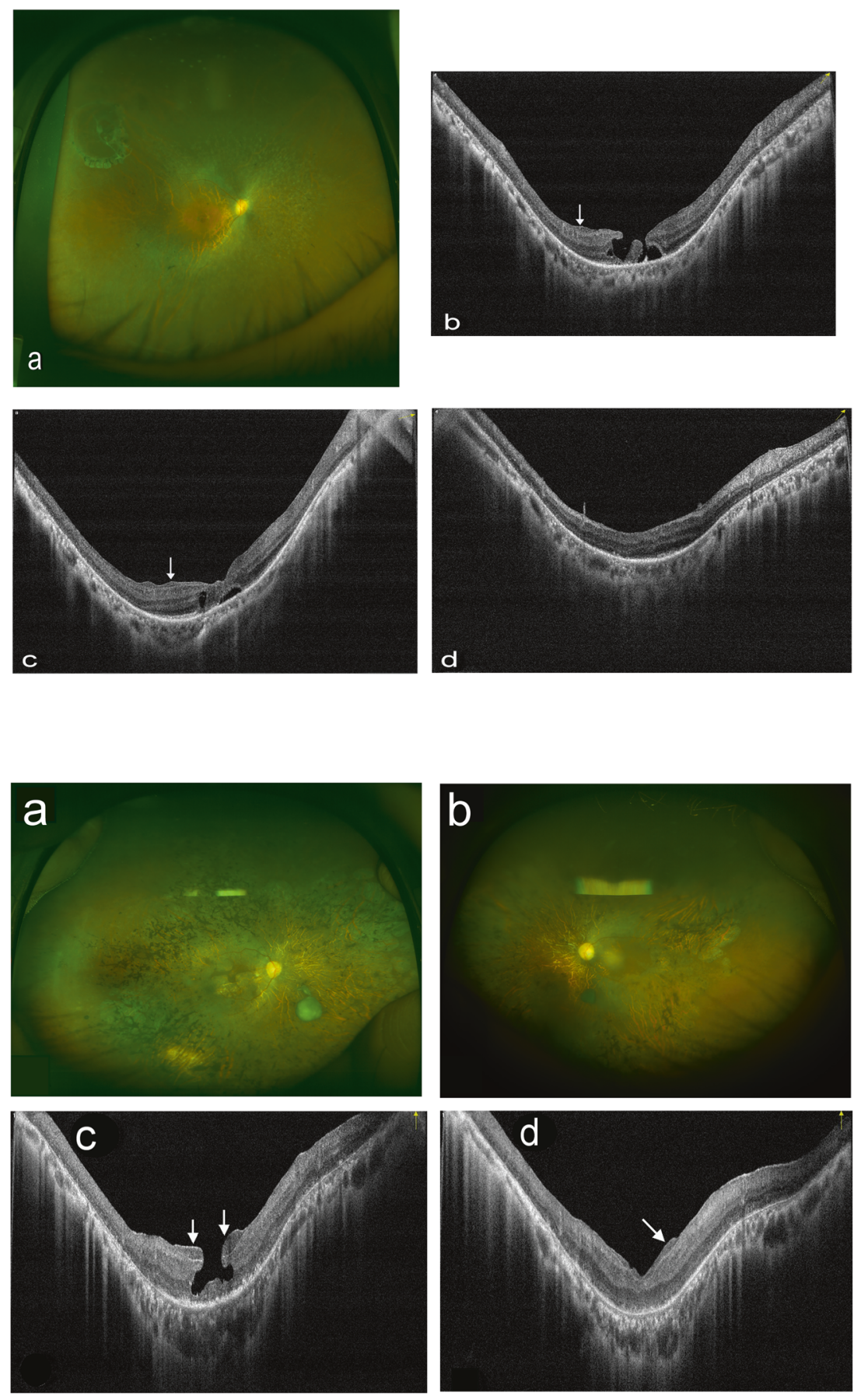

Fig. 3 a, b Ultra-wide scanning laser ophthalmoscopy (UWSLO) demonstrates peripheral bone spicule pigmentation in both eyes of patient 3. c Spectral-domain optical coherence tomography (SD-OCT) images show

lamellar macular hole (LMH) with wide intraretinal defects and foveal bump in the left eye. Lamellar hole-associated epiretinal membrane (LHEP) (arrows) was also detected. d Three years later, OCT image shows the LMH were closed in the left eye. LHEP remained (arrow) as detected by en face OCT imaging, with reduced central retinal thickness; further, VA increased.

Condensed posterior hyaloid and vitreoretinal adhesion were found in the right eye of patient no. 6 on OCT. Vitreous degeneration is common in RP eyes and it could lead to vitreous collapse, followed by posterior vitreoschisis, which has been reported prior to the formation of atypical posterior vitreous detachment that includes condensed irregular fibre aggregates (as observed in this eye) [21]. Therefore, the LMH in this eye may be caused by vitreous degeneration and 
vitreous traction. This eye with CMO experienced a spontaneous closure of the LMH during follow up. CMO resolve months later. However, VA did not improve, which may be the result of the long-standing CMO. The presence of a foveal bump was noted in six $(60 \%)$ LMH eyes. On OCT, five (83.3\%) of these eyes demonstrated a combination of wide intraretinal defects, this is similar to the description of degenerative LMHs by Govetto et al. [7]. It is unclear whether the formation of degenerative LMH is directly triggered by retinal degeneration or indirectly by vitreomacular traction that enhances the deterioration in these eyes; thus, this should be further studied. For these eyes, one eye experienced a spontaneous closure of the LMH during follow up and no improvement in VA was found. PPV was performed in one eye, which was accompanied by ERM on OCT imaging. After surgery, sealing of LMH and improving VA were achieved after surgery.

The pathogenesis of MHs in RP patients remains unclear. In our cohort, one eye with FTMH exhibited extensive and highly elevated macular detachment, one eye with LMH was complicated with extensive and highly elevated macular retinoschisis, and the other eyes demonstrated only foveal area changes, such as intraretinal defects or foveal bump. The difference observed here may because they arose from different pathogenic mechanisms traction or degeneration. Eyes had more extensive changes may be caused by tractional forces and eyes had just foveal area changes may be caused by progressive retinal degeneration. According to the above clinical studies, the formation of MHs in these eyes might be multifactorial, rather than mono-factorial. Factors that may contribute to the formation of LMH in RP eyes include high myopia, macular schisis, vitreous degeneration, traction of condensed vitreous strands or ERM, and chronic CMO. In addition, high prevalence of LHEP in these eyes maybe due to the severe disruption of retinal tissue, which stimulate glial cell proliferation. Moreover, vitreous changes and complex vitreoretinal interactions that are present in these RP eyes may also play a part.

Surgical intervention for MH in RP remains controversial because it generally does not reverse poor visual function or slow disease progression. In addition, since OCT images indicate that LHEP is connected to the remaining central foveal tissue, there is a fear of subsequent FTMH formation if it is peeled [3]. However, LMHs with LHEP have shown significant morphological progression, as well as enlargement of both LHEP area and hole diameter during long-term follow-up [22]. Further, eyes that underwent surgery in our study demonstrated complications of retinal detachment, highly elevated retinoschisis, ERM, and CMO. Therefore, PPV was performed along with ILMP. During the operation, we did not forcefully remove the yellowish LHEP tissue around the hole margin; instead, we introduced LHEP tissue into the $\mathrm{MH}$ carefully. Postoperative examination demonstrated anatomical recovery and increased VA in all eyes. No eyes developed FTMH during follow up. The current surgery might have resulted in favourable outcomes because the procedure relieved tractional forces and removed residual vitreous that was present on the retinal surface. Further, the modified procedure, which included preservation of hole margin LHEP, aided in the closure of MHs. Importantly, we suspect that sealing these holes may prevent progressive retinal tissue loss due to MHs. In addition, spontaneous closure of MHs was documented in the current study. Spontaneous closure was found in two eyes, which may be due to the LHEP tissue migration that was reported before [11]. Interestingly, one patient demonstrated LMH and CMO in both eyes in our cohort. The left eye obtained sealed hole after surgery and the right eye experienced spontaneous hole closure without any procedures. Improved VA was documented in the left eye but not in the right eye. We speculate that the damage caused by the long duration of macular oedema played a significant role because it costed more time for the retinal oedema of right eye to resolve than that of the left eye.

The current study presents limitations related to its retrospective design. Furthermore, we were only able to enrol a limited number of patients in the study because the formation of MHs is rare in RP patients. Therefore, we could not draw strong conclusions. However, we explored the clinical findings of MHs in RP and, for the first time, reported a high incidence of LHEP in these eyes, which could help clinicians to better understand the pathogenesis of macular abnormalities in RP. The surgical outcomes in our study may also help to guide clinicians in management of this condition.

In conclusion, we used OCT images to identify a specific type of epiretinal proliferation, LHEP, that was commonly found in MHs that formed in RP eyes; we confirmed the presence of LHEP during surgery. To our knowledge, this is the first study to explore the associations between LHEP and MHs in RP patients. In addition, we showed that surgical repair can be successful in improving macular morphology, but improvements in visual function may be limited because of the long-standing retinal dysfunction and disease progression in RP eyes. Therefore, this guarded prognosis should be discussed with the patients before the surgery.

\section{Summary}

\section{What was known before}

- MH formation is infrequent in eyes with RP.

- Occurrence of MHs may seriously damage central vision. 


\section{What this study adds}

- In this study, LHEP was commonly found in the eyes of RP patients with MHs, which was not reported before.

- In addition, this study reported anatomical and functional improvements of these eyes after a conservative vitreoretinal surgery.

- This study also documented spontaneous closure in two eyes, which showed no gain of VA.

- This study tried to explore the relationship between LHEP and MHs in RP for the first time.

Authors contributions Design and conduct of the study (JL and PZ); collection, analysis, and interpretation of data (JL, JL, and XZ); preparation of the manuscript (JL); and critical review and final approval of the manuscript (PZ).

\section{Compliance with ethical standards}

Conflict of interest The authors declare that they have no conflict of interest.

Ethics approval and consent to participate This study was approved by Ethics Committee of Xin Hua Hospital affiliated to Shanghai Jiao Tong University School of Medicine. All procedures performed in studies involving human participants were in accordance with the ethical standards of the institutional and/or national research committee and with the 1964 Helsinki Declaration and its later amendments or comparable ethical standards.

Informed consent Informed consent was obtained from all individual participants included in the study. Informed consent was obtained from all individual participants to publish the study.

Publisher's note Springer Nature remains neutral with regard to jurisdictional claims in published maps and institutional affiliations.

\section{References}

1. Hartong DT, Berson EL, Dryja TP. Retinitis pigmentosa. Lancet. 2006;368:1795-809.

2. Jin ZB, Gan DK, Xu GZ, Nao IN. Macular hole formation in patients with retinitis pigmentosa and prognosis of pars plana vitrectomy. Retina. 2008;28:610-4.

3. Parolini B, Schumann RG, Cereda MG, Haritoglou C, Pertile G. Lamellar macular hole: a clinicopathologic correlation of surgically excised epiretinal membranes. Invest Ophthalmol Vis Sci. 2011;52:9074-83.

4. Ozdek S, Ozdogan S, Sezgin T, Gurelik G. Bilateral disc edema and unilateral macular hole in a patient with retinitis pigmentosa. Eur J Ophthalmol. 2006;16:487-90.

5. Giusti C, Forte R, Vingolo EM. Clinical pathogenesis of macular holes in patients affected by retinitis pigmentosa. Eur Rev Med Pharm Sci. 2002;6:45-8.
6. Pang CE, Spaide RF, Freund KB. Comparing functional and morphologic characteristics of lamellar macular holes with and without lamellar hole-associated epiretinal proliferation. Retina. 2015;35:720-6.

7. Govetto A, Dacquay Y, Farajzadeh M, Platner E, Hirabayashi K, Hosseini $\mathrm{H}$, et al. Lamellar macular hole: two distinct clinical entities? Am J Ophthalmol. 2016;164:99-109.

8. Rino F, Elena Z, Ivan M, Paolo B, Barbara P, Federica R. Lamellar macular hole in high myopic eyes with posterior staphyloma: morphological and functional characteristics. Graefes Arch Clin Exp Ophthalmol. 2016;254:2141-50.

9. Vingolo EM, Valente S, Gerace E, Spadea L, Nebbioso M. Macular hole in retinitis pigmentosa patients: microincision vitrectomy with polydimethylsiloxane as possible treatment. Eye. 2015;29:699-702.

10. Rush RB, Rush SW. Bilateral lamellar macular hole surgery in retinitis pigmentosa. Retin Cases Brief Rep. 2016;10:83-5.

11. Raja M, Goldsmith C, Burton BJ. Spontaneous resolution of fullthickness macular hole in retinitis pigmentosa. BMJ Case Rep. 2011;2011:bcr0320113999. https://doi.org/10.1136/bcr.03. 2011.3999.

12. Hagiwara A, Yamamoto S, Ogata K, Sugawara T, Hiramatsu A, Shibata M, et al. Macular abnormalities in patients with retinitis pigmentosa: prevalence on OCT examination and outcomes of vitreoretinal surgery. Acta Ophthalmol. 2011;89:e122-5.

13. Testa F, Rossi S, Colucci R, Gallo B, Di Iorio V, della Corte M, et al. Macular abnormalities in Italian patients with retinitis pigmentosa. Br J Ophthalmol. 2014;98:946-50.

14. Witkin AJ, Ko TH, Fujimoto JG, Schuman JS, Baumal CR, Rogers $\mathrm{AH}$, et al. Redefining lamellar holes and the vitreomacular interface: an ultrahigh-resolution optical coherence tomography study. Ophthalmology. 2006;113:388-97.

15. Pang CE, Spaide RF, Freund KB. Epiretinal proliferation seen in association with lamellar macular holes: a distinct clinical entity. Retina. 2014;34:1513-23.

16. Compera D, Entchev E, Haritoglou C, Scheler R, Mayer WJ, Wolf $\mathrm{A}$, et al. Lamellar hole-associated epiretinal proliferation in comparison to epiretinal membranes of macular pseudoholes. Am J Ophthalmol. 2015;160:373-84.e1.

17. Amemiya K, Takahashi M, Nishida A, Matsumura M, Hayakawa M, Honda Y. [A macular hole in the eye of a young patient with retinitis pigmentosa]. Nippon Ganka Gakkai Zasshi. 2002; 106:236-42.

18. Baba T, Ohno-Matsui K, Futagami S, Yoshida T, Yasuzumi K, Kojima A, et al. Prevalence and characteristics of foveal retinal detachment without macular hole in high myopia. Am J Ophthalmol. 2003;135:338-42.

19. Wang N-K, Wu Y-M, Wang J-P, Liu L, Yeung L, Chen Y-P, et al. Clinical characteristics of posterior staphylomas in myopic eyes with axial length shorter than 26.5 millimeters. Am J Ophthalmol. 2016;162:180-90.e1.

20. Lai TT, Yang CM. Lamellar hole-associated epiretinal proliferation in lamellar macular hole and full-thickness macular hole in high myopia. Retina 2017;38:1316-23.

21. Takezawa M, Tetsuka S, Kakehashi A. Tangential vitreous traction: a possible mechanism of development of cystoid macular edema in retinitis pigmentosa. Clin Ophthalmol. 2011;5:245-8.

22. Compera D, Schumann RG, Cereda MG, Acquistapace A, Lita V, Priglinger SG, et al. Progression of lamellar hole-associated epiretinal proliferation and retinal changes during long-term follow-up. Br J Ophthalmol. 2017;102:84-90. 